\title{
A CONCEPT ON IMPLEMENTATION OF SMART CITY
}

\author{
Aman Patel, Bharat, Akaanksha Gupta, Sonika Singh \\ Department of CSE \\ IMS Engineering College, Ghaziabad, U.P., India \\ Mr. Atul Kumar \\ Department of CSE \\ IMS Engineering College, Ghaziabad, U.P., India
}

\begin{abstract}
As higher number of population is living in metro cities, hence it's important to make the cities smarter for their movement and accommodation. Smart refers to the intellectual use of technology to improve the productivity of a city's essential infrastructure. Smart City removes the inferior complexity of our daily lives and improve the standard of life of inhabitants in these cities. Smart City is moreover considered as a measure of intelligence in urban areas. We put the features of a city that are pre-requisite to be considered as a smart city. This paper focus is on the challenge to minimize language barriers for any tourist or inhabitant of a smart city. Any person new to a smart city must be able to easily mobilize around the city with the help of advanced technologies. We try to take advantage of already established technologies to implement in a more strategic, centralized and integrated manner to benefit locals, tourists, governments, businesses, etc.. The device that is present with us most of the time is smart phone and there is a valuable network among mobile services by the central and local government. Therefore, the services provided via smart phones like apps will help the citizens to make the best use of services provided in a smart city.
\end{abstract}

Keywords - Application, Android, Smart City.

\section{INTRODUCTION}

Nowadays, people like to explore new things in their daily life, especially new cities or even hidden qualities and gems of their own city. While exploring new cities people face many issues like what to see in the city, what's happening in the city in the current scenario, what's unique in the city, and how to communicate in the local language, etc. Also the people already living in these cities are interested in utilizing its facilities and services in a more efficient manner. Therefore it's very crucial to develop solutions to such problems in the real time. The solutions based on technological advancement are the best approach to handle such problems. If we try to optimize and integrate the solutions already available in an efficient manner then it can result in one of the best possible solutions to any such problem.

Smart city is one such solution to the above discussed problems. There are various other models of smart cities already effective in various parts of the world. But none of them has engaged the user that is the local people and the tourists visiting the city in a very effective and seamless manner. Hence, there's a huge gap between the local administration and the inhabitants of the city. We want to establish a technology driven solution to make cities smarter and the services provided to citizens in a centralized and efficient manner. Therefore, we chose to make use of the most widely used technology that comes in handy with everyone and that's smart phones. Smart phones are incorporated with various technologies like apps which are the core of user experience. We made an application "Smart City" using android as frontend and PHP as backend for the smart phone users in the city. It's an application that uses services of GPS, contacts, web scrolling, etc to enhance the features of smart city app like nearby places, famous places, weather forecast, current and updated news, social and local groups, events happening in the city, language translation, etc. The application is build in android SDK as it's rightly effective in the development of app and user friendly for both the user and the programmer. With the use of app the gap between the services and information provided by the city administration and the locals and the visitors will reduce immensely.

\section{PROPOSED WORK}

Development of self driving cars and intelligent transportation systems have shown a growth evolution in the last decade. Road sign detection and identification is the most challenging task confronted by the researchers and developers. Road sign detection includes two steps. First is the feature extraction and second is the signs recognition. In the first step, many methods had been proposed like scale invariance feature, edge detection, histogram of gradient and others. Many algorithms are used like SVM, KNN classifier, artificial neural network etc. Currently traditional computer vision algorithms are gradually replaced 


\section{International Journal of Engineering Applied Sciences and Technology, 2020 \\ Vol. 5, Issue 1, ISSN No. 2455-2143, Pages 403-407 \\ Published Online May 2020 in IJEAST (http://www.ijeast.com)}

by the different applications like object classification and pattern recognition.

To implement smart city as an application for public use we use the following technologies:-

\section{Android Studio}

Android Studio is the official integrated development environment (IDE) for Google's Android operating system, built on JetBrains' IntelliJ IDEA software and designed specifically for Android development. It is available for download on Windows, macOS and Linux based operating systems. It is a replacement for the Eclipse Android Development Tools (ADT) as the primary IDE for native Android application development [1].

\section{Server Side Language}

- Server side language is used in web development that performs tasks on the server.[2]

- It employs scripts on a web server which produce a response customized for each user's request to the website [3].

\section{Text Translation}

For text translations we used here Google API's in this project like Google translator for translating languages and text to speech engine for converting the text retrieved into the audio. Google APIs is a set of application programming interfaces (APIs) developed by Google which allow communication with Google Services and their integration to other services. Google Translate can translate multiple forms of text and media, including text, speech, images, sites, or real-time video, from one language to another. Here we sequence to sequence model whose algorithm is explained below:-

\section{Summary of the algorithm}

- We start with input sequences from a domain (e.g. English sentences) and corresponding target sequences from another domain (e.g. French sentences).

- An encoder LSTM turns input sequences to 2 state vectors (we keep the last LSTM state and discard the outputs)

- A decoder LSTM is trained to turn the target sequences into the same sequence but offset by one timestep in the future, a training process called "teacher forcing" in this context. It uses as initial state the state vectors from the encoder. Effectively, the decoder learns to generate targets $[\mathrm{t}+1 \ldots]$ given targets $[\ldots \mathrm{t}]$, conditioned on the input sequence.

- In inference mode, when we want to decode unknown input sequences, we:

○ Encode the input sequence into state vectors
- Start with a target sequence of size 1 (just the start-of-sequence character)

- Feed the state vectors and 1-char target sequence to the decoder to produce predictions for the next character

- Sample the next character using these predictions (we simply use argmax).

- Append the sampled character to the target sequence

- Repeat until we generate the end-of-sequence character or we hit the character limit [4].

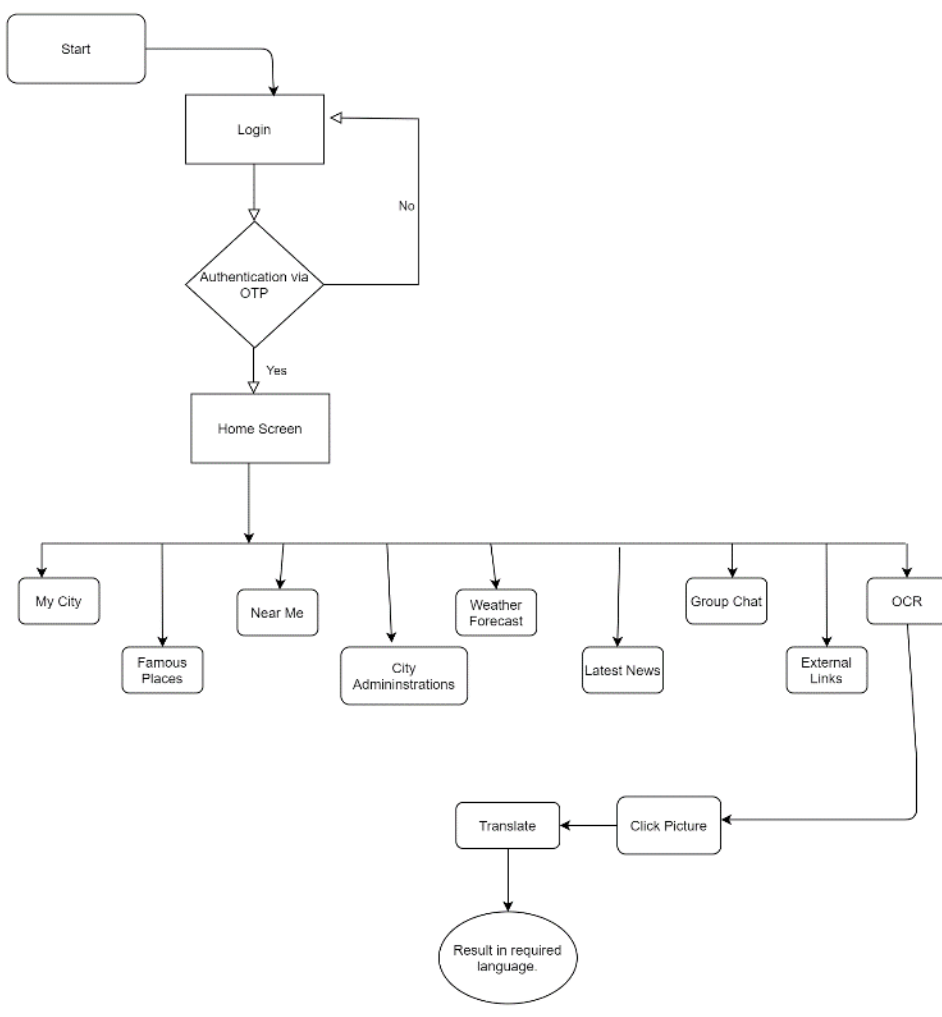

Figure 1. Flow Chart

Figure 1 is flowchart of our system which shows flow of our Smart City application.

\section{EXPERIMENT AND RESULT}

Based on the above research and its implementation we have acquired the following results:- 
International Journal of Engineering Applied Sciences and Technology, 2020

Vol. 5, Issue 1, ISSN No. 2455-2143, Pages 403-407

Published Online May 2020 in IJEAST (http://www.ijeast.com)

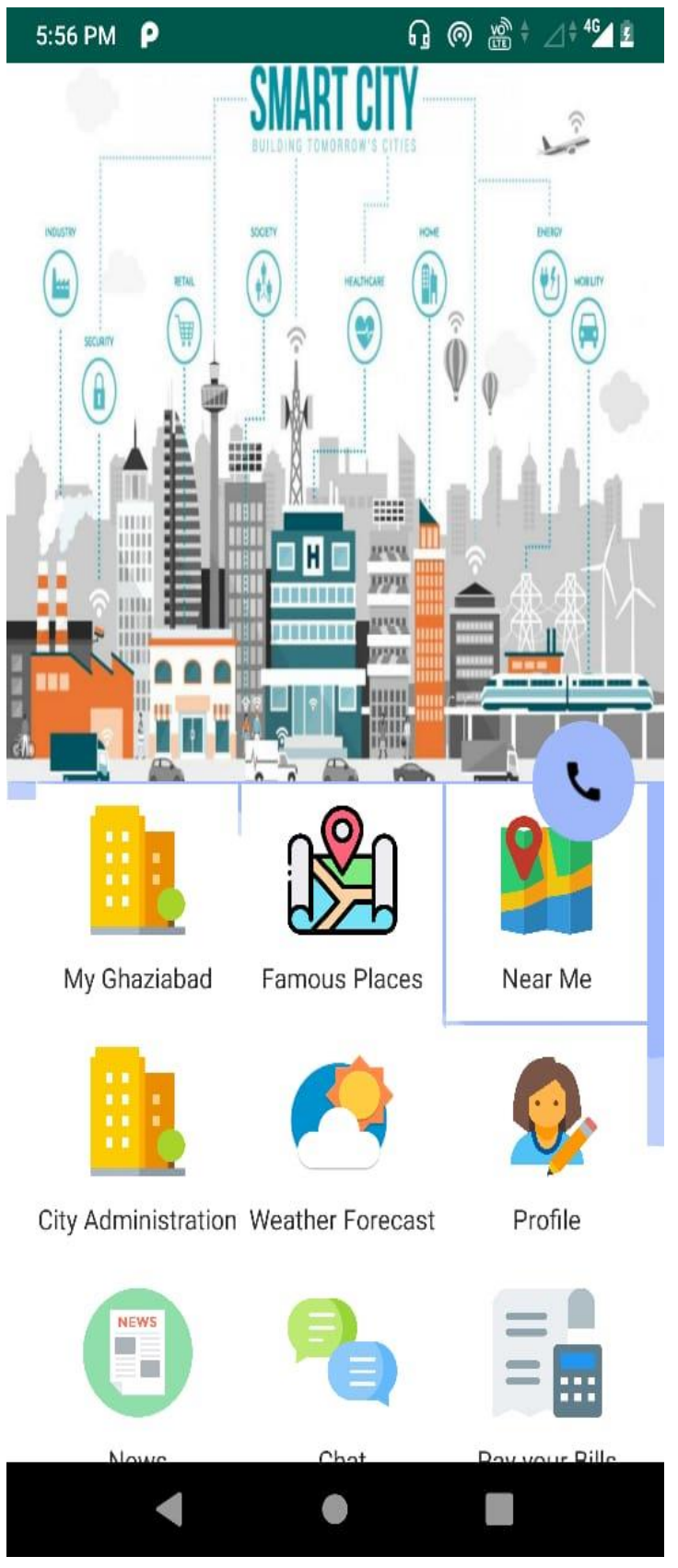

Figure 2. Screenshot of home page of smart city app

Figure 2 shows the home page which guides the user through the various aspects of the app and its main features.

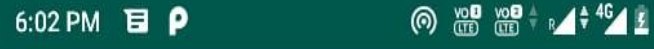

\section{Please Select Group you want to join}

Please select any group

Swach Bharat

Tourism

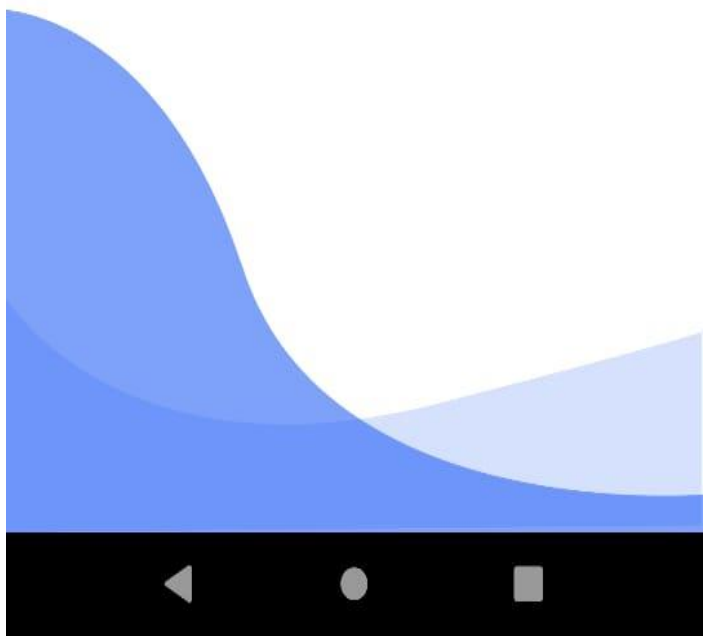

Figure 3. Screenshot of the app smart city for SignIn.

Figure 3 explains the communication enhancement achieved through the smart city app. This way user can take advantage of the various happenings in the city thus leading to user engagement with the app and the smart city. 
International Journal of Engineering Applied Sciences and Technology, 2020

Vol. 5, Issue 1, ISSN No. 2455-2143, Pages 403-407

Published Online May 2020 in IJEAST (http://www.ijeast.com)

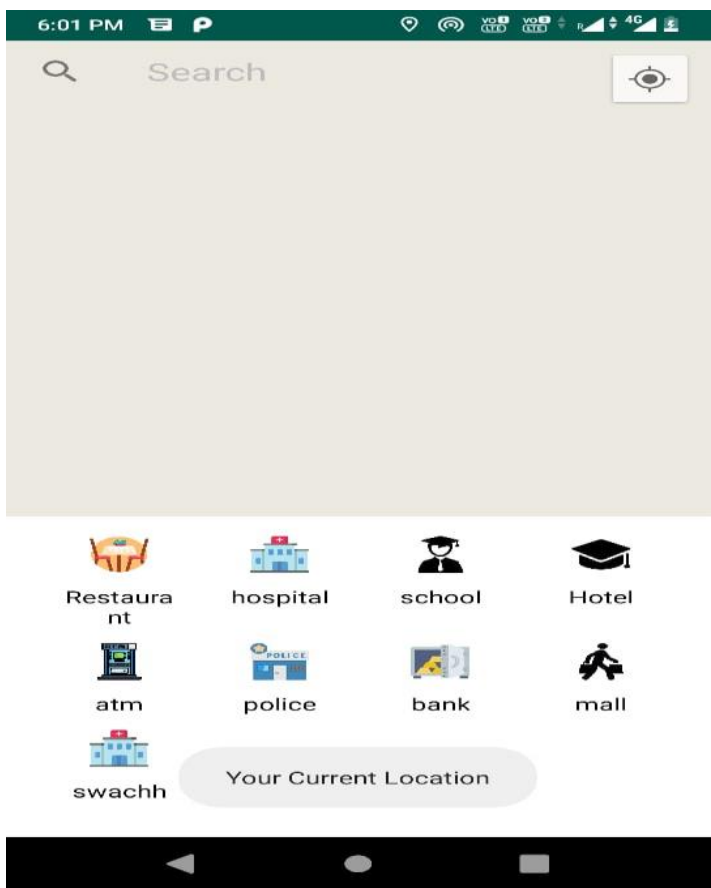

Figure 4. Screenshot of the smart city app for Near Me.

Figure 4 shows the nearby places available to a particular user in the city which enables smoothing of mobility in the city.
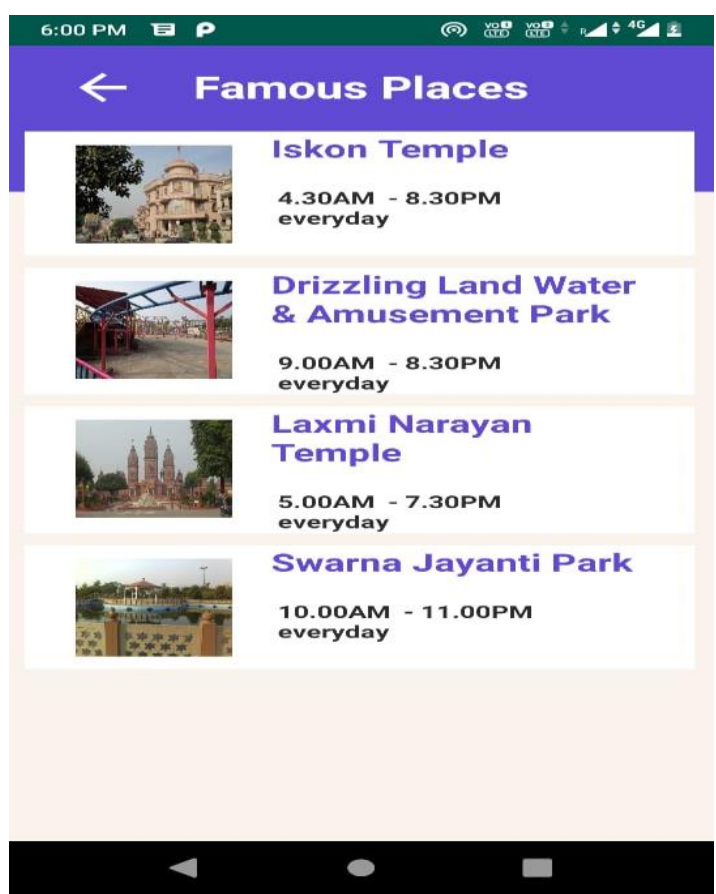

Figure 5. Screenshot of the smart city app for Famous Places.

Figure 5 clearly depicts the famous places present in the city for visitors and the locals.

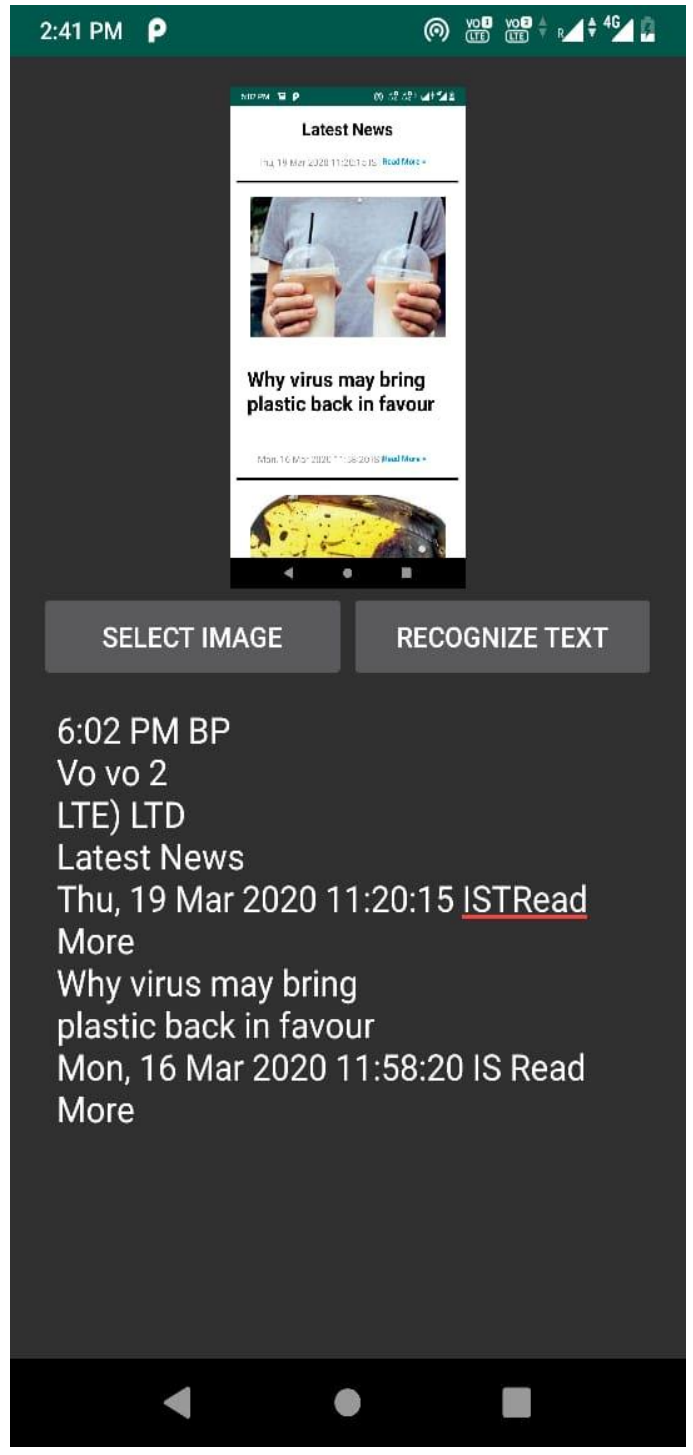

Figure 6. Screenshot of the smart city app for OCR.

Figure 6 shows the Language translation obtained through the Google API's and OCR technologies.

\section{CONCLUSION}

We have proposed an efficient and dependable android smart city utility. The initiation was taken to benefit the human beings to ease their work in the city. This application will ease the mobility in the city of any visitor and locals present in the city. This application also helps in bridging the gaps between the language commuted by the visitor and the locals in the city. The sign boards and various writing in the city will be easily readable by the user. We have tried to ensure ease of user experience for anyone who uses the application. Smart city is here to stay and expand its features to build a sustainable city for both people and environment. The solution we provided is cost-efficient and 


\section{International Journal of Engineering Applied Sciences and Technology, 2020 \\ Vol. 5, Issue 1, ISSN No. 2455-2143, Pages 403-407 \\ Published Online May 2020 in IJEAST (http://www.ijeast.com)}

strategic for many purposes. It will help in improving the infrastructure of the city. There will be increment in business as people will be more engaged with the events happening in the city.

\section{ACKNOWLEDGEMENTS}

Our sincere thanks to guide Mr. Atul Kumar, Associate Professor, IMS Engineering College, Ghaziabad, India for his patient guidance and constructive suggestions for the research in the area of android application development. This research is made possible due to her willingness to devout time generously.

\section{REFERENCE}

[1] Iversen and Eierman M.. (2013). Learning Mobile App Development. Pearson Education, Inc, pp. 1-350.

[2] Singhal A..(2010). Location -Based Mobile App for Android Platform, 1st ed. Austin: University of Texus, pp. 5-95.

[3] Shu H.. (2010). City Guide over Android, 1st ed. Norway: Norweigian University of Science and Technology, pp. 595

[4] Hochreiter S. and Schmidhuber J..(1997). "Lstm can solve hard long time lag problems," in Advances in neural information processing systems,pp. 473-479

[5] Rumelhart D. , Hinton G. E., and Williams R. J.(1986). Learning representations by back-propagating errors. Nature, 323(6088):533-536,

[6] Mikolov T., Sutskever I., Chen K., Corrado G. S., and Dean J.(2013) "Distributed representations of words and phrases and their compositionality," in Advances in neural information processing systems, pp. 3111-3119.

[7] Wallace R. S..(2009). "The anatomy of alice," in Parsing the Turing Test. Springer, pp. 181-210.

[8] Pennington J., Socher R., and Manning C.(2014). "Glove: Global vectors for word representation," in Proceedings of the 2014 conference on empirical methods in natural language processing (EMNLP), 2014, pp. 1532-1543.

[9] Jain K. and Yu B..(1998). "Automatic Text Location in Images and Video Frames," International Conference of Pattern Recognition (ICPR), Brisbane, pp. 1497-1499.

[10] Q Ye, Doermann D..(2015). Text detection and recognition in imagery: A survey. IEEE transactions on pattern analysis and machine intelligence.37(7):1480-500.

[11] Trier ØD, Jain AK, Taxt T..(1996). Feature extraction methods for character recognition-a survey. Pattern recognition. 29(4):641-62.

[12] Eikvil Line.(1993). "Optical Character Recognition", NorskRegnesentral, Oslo, Norway, Rep. 876. 